\title{
Quantum entanglement in astrophysics
}

\author{
Javier Gomez* ${ }^{\dagger}$ \\ The Citadel \\ E-mail: javier.gomez@citadel.edu
}

\begin{abstract}
We found that the most feasible naturally produced quantum entanglement between two distant particles from astrophysical regions is the two-photon spontaneous transition of the hydrogen $2{ }^{2} S_{1 / 2}$ metastable level, which is known to be one of the components of the continuous spectra of ionized regions. We calculate the two-photon emission rate the Orion Nebula, two nearby planetary nebulae IC 2149 and NGC 7293, and the Solar Corona; whose production of entangled pairs per second is $5.80 \times 10^{48}, 9.39 \times 10^{45}, 9.77 \times 10^{44}$, and $1.46 \times 10^{16}$ respectively. The distribution of the propagation directions of both emitted photons does not vanish at any angle; therefore it is possible to observe the entangled pair at an angles $\theta \approx 0^{\circ}$. Because the number of two-photon coincidences goes as the fourth power of the ratio between the detector size and the distance from the astrophysical object, coincidences are scarce; for its detection we required receivers with a size much larger than those currently available.
\end{abstract}

Quantum of Quasars workshop - QQ09,

December 2-4, 2009

Grenoble, France

\footnotetext{
*Speaker.

${ }^{\dagger}$ Based on the paper: J. Gomez, A, Peimbert, \& J. Echevarría, Optical Quantum Entanglement in Astrophysics, $R M x A A 45$ (179); thanks to the Instituto de Astronomía UNAM, México, where most of this paper was done.
} 


\section{Introduction}

I discuss the methods and results of our paper ${ }^{[10]}$ which goal is to connect the optical quantum entanglement with astrophysics. Since the publication of the Einstein, Podolsky, \&Rosen ${ }^{[7]}$ paper in 1935 which was though to criticized the fundamentals of Quantum Mechanics, and point the finger on its incompleteness; it allows however, the introduction of the quantum entanglement between two separated particles. In this presentation I am going to discuss the most feasible kind of quantum entanglement: the two-photon emission in the hydrogen $2{ }^{2} S_{1 / 2}$ metastable level which is one of the most important component in the optical continuum emission from HII regions, and planetary nebulae; we calculate the emission rate for 4 different astrophysical regions and the possibility to detecting the entangled pairs of photons.

\section{Quantum entanglement}

One of the physicist dreams is to be able to communicate to distant points in the Universe; and above all to allow the communication of quantum information between to distant points of the Universe. The quantum entanglement came to life from the famous paper of Einstein, Podolsky, \& Rosen $^{[7]}$, with a Gedanken Experiment of a quantum composite state that can not be expressed as a product of the two separate states of each particle, therefore they are in an entangled state. This presented new points of view about realism and non-locality in QM. Later on Bohm ${ }^{[3]}$ (1951) used discrete states in a case of quantum entanglement with the description of particle's spins; which is going to be used by John Bell ${ }^{[2]}$, who develops a general model of hidden variables, where the angular momentum is conserved, as in the case of SPS atomic cascades, where it results some algebraic inequalities of the involved values of this hidden variables model. The Bell's theorem is precisely referred to this general hidden variables model, under the assumptions of locality, i.e., that there is no instantaneous influence or perturbation between the separated particles and the values should obey certain algebraic inequality which is totally independent of QM. Several experiments ${ }^{[6]}$ were done to confirm the violation of a special kind of Bell's inequality that does not required polarization analyzers with $100 \%$ efficiency, named $\mathrm{CHSH}$ (Clauser-Horne-ShimonyHolt) Bell's inequality; and finally with the experiment of Aspectetal. ${ }^{[1]}$, where they measured the linear polarization correlations of pairs of photons with time-varying analyzers; for their solid angles and polarizer efficiencies, QM predicts $S_{Q M}=0.112$, (the $S$ variable is defined therein). They found that the average of their two runs, yields $S_{\exp }=0.101 \pm 0.020$ violating the ShimonyHolt inequality $S \leq 0$ (an equivalent of Bell's inequality) by 5 standard deviations, which effectively disproved the existence of local hidden variables theories.

These are examples of quantum entanglement that can be performed at the Lab: positronium annihilation, proton-proton scattering, decay of $\pi^{0} \rightarrow e^{+}+e^{-}$, cascade-photon experiments (i.e. $J=0 \rightarrow J=1 \rightarrow J=0$ ), and two-photon experiments of the hydrogen metastable forbidden transition $2{ }^{2} S \rightarrow 1{ }^{2} S^{[9][4]}$.

Among all the possible sources of naturally produced quantum entanglement in the Universe: the annhilation of the positronium, which produces a pair of entangled $\gamma$ photons (a process that occurs spontaneously in stellar interiors, stellar coronae, or cosmic rays), the decay of a spinless particle $\pi^{0} \rightarrow e^{+}+e^{-}$(happens in some nuclear reactions in stellar interiors or in cosmic rays), 


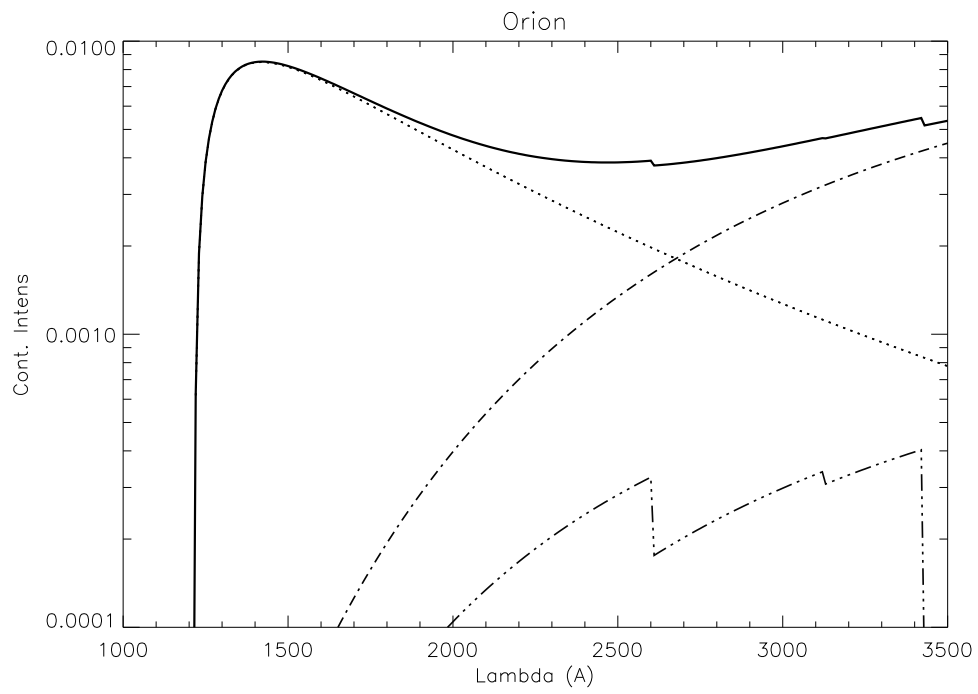

Figure 1: Continuum emission of Orion Nebula, where ...: two-photon, _._.: recombination of HI, _......: recombination of $\mathrm{HeI} \&$ : total. C. Morisset(Inst.Astro.UNAM).

the proton-proton scattering (may occur in stellar atmospheres or in the ISM), the two-photon atomic SPS cascades such as the case of observed $\lambda 5513 \AA$ and $\lambda 4227 \AA$ pairs produced in the Calcium atom (may occur in stellar atmospheres), and the two-photon emission in the hydrogen $2{ }^{2} S_{1 / 2}$ metastable level that occurs in large quantities in HII regions and PNe (is observable as part of their optical whole continuum emission). From all of these kinds of pairs of quantum entangled particles, the most feasible to be detected is the two-photon emission in the hydrogen $2{ }^{2} S_{1 / 2}$ metastable; due to the abundance of hydrogen in the universe and to the moderate amount of energy involved in his process.

\subsection{Two-photon emission in the hydrogen $2{ }^{2} S_{1 / 2}$ metastable}

The continuum emission coefficients in HII Regions \& PNe can be expressed in four components: free-bound \& free-free transitions of $\mathrm{H}+$, free-bound $\&$ free-free transitions of $\mathrm{He}$, freebound \& free-free transitions of $\mathrm{He}+$, and $2{ }^{2} S_{1 / 2}$ two-photon transitions in $\mathrm{H}$.

As is shown in figure 1, for the case of Orion most of the continuum is due two-photon emission for $\lambda<2600 \AA$.

Novick $^{[13]}$ (1969) presented an extensive review of the previous work on two-photon productions, and showed (both theoretically and experimentally) that the angular correlation between the propagation directions between both photons is proportional to $\left(1+\cos ^{2} \theta\right)$, which clearly shows that both photons can be emitted in all directions with respect to each other. This is an important result since for astrophysical detection it is convenient that the direction of propagation of the two-photon pair has to be nearly parallel.

Two-photon entangled wavefunction in the polarizations can be expressed in this way: 


$$
\Psi=\frac{1}{\sqrt{2}}\left[\left(\begin{array}{l}
1 \\
0
\end{array}\right) \otimes\left(\begin{array}{l}
1 \\
0
\end{array}\right)+\left(\begin{array}{l}
0 \\
1
\end{array}\right) \otimes\left(\begin{array}{l}
0 \\
1
\end{array}\right)\right]
$$

Where $\left(\begin{array}{l}1 \\ 0\end{array}\right)$ represents the polarization in the direction of $\hat{x}$ axis \& $\left(\begin{array}{l}0 \\ 1\end{array}\right)$ represents the polarization in the direction of $\hat{y}$ axis; first column of tensor product $\otimes$ is the state of the first photon and the second column of the tensor product $\otimes$ is the state of the second photon.

The conservation of energy of two-photon in terms of frequencies:

$$
v_{s}+v_{i}=v_{21}=\frac{E_{2}-E_{1}}{h}=\frac{10.2 \mathrm{eV}}{h}=1.46 \times 10^{15} \mathrm{~s}^{-1}
$$

With a total transition probability: $A_{2^{2} S, 1^{2} S}=8.227 \mathrm{sec}^{-1}$.

\section{Astrophysical ionized regions}

Here I present the methods to calculate the total number of entangled emitted photons: $\mathbf{Q}(\mathbf{2 q})$, and the total number of emitted photons produced by the object via the hydrogen metastable transition decay $2{ }^{2} S \rightarrow 1{ }^{2} S: \mathbf{Q}\left(\mathbf{H}^{\mathbf{0}}\right)$ production rate of photoionizing photons.

The astrophysical regions that we have studied are:

- PNe: IC 2149 (L=1 585 pc) NGC 7293[Helix] (L=157 pc)

$$
Q\left(H^{0}\right)=\frac{\alpha_{B}\left(H^{0}, T\right)}{\alpha_{H_{\beta}}^{\text {eff }}\left(H^{0}, T\right)} \times \frac{L\left(H_{\beta}\right)}{h v_{H_{\beta}}}=\frac{\alpha_{B}}{\alpha_{H_{\beta}}^{\text {eff }}} \times \frac{\pi F\left(H_{\beta}\right)}{4 \pi L^{2} h v_{H_{\beta}}},
$$

$Q\left(H^{0}\right)$ obtained from $H \beta$ flux: $Q(2 q)=\xi \cdot Q\left(H^{0}\right), \xi=\frac{2 S}{1+n_{e} r}$.

$Q(2 q)\left[\right.$ IC 2149] $=1.88 \times 10^{46}$ photons s $^{-1}$

$Q(2 q)[N G C 7293]=1.95 \times 10^{45}$ photons s$^{-1}$

- Orion $\operatorname{Nebula}(\mathrm{L}=414 \mathrm{pc})$

Dominant O6 star $\theta^{1}$ Ori C

$Q\left(H^{0}\right)=2.19 \times 10^{49}$ photoionizing photons $\mathrm{s}^{-1}$,

$\xi=0.53$

$Q(2 q)=1.16 \times 10^{49}$ photons s $^{-1}$

- Solar Corona (L=less than 1 A.U.)

$$
\begin{aligned}
Q(2 q)= & 2 \int_{0.5}^{1} \int_{0}^{4 \pi} \int_{\zeta=1}^{\zeta=\zeta_{l}} \frac{4 \pi R_{\odot}^{3}}{4 \pi h v} \\
& \times F_{e}(\zeta) \times\left(\frac{\zeta^{2} n_{e}^{2}(\zeta) \alpha_{B}(\zeta)}{A_{2^{2} S, 1^{2} S}} \times h y A_{2 p}(y)\right) d y d \Omega d \zeta \\
& =2.92 \times 10^{16} \text { photons } \cdot \mathrm{s}^{-1}
\end{aligned}
$$


Table 1: Receiver diameters for one two-photon coincidence per hour and per year

\begin{tabular}{l|llrrr}
\hline \hline Object & $\mathrm{L}$ & $\begin{array}{l}R_{P P} \\
\text { pairs s }^{-1}\end{array}$ & $\begin{array}{r}D[1 / y]^{*} \\
\mathrm{~km}\end{array}$ & $\begin{array}{r}D[1 / y] \\
\mathrm{km}\end{array}$ & $\begin{array}{r}D[1 / h r] \\
\mathrm{km}\end{array}$ \\
\hline Orion Nebula & $414 \mathrm{pc}$ & $5.8 \times 10^{48}$ & 272 & 785 & 7590 \\
IC 2149 & $1585 \mathrm{pc}$ & $9.39 \times 10^{45}$ & 5200 & 14000 & 135000 \\
NGC 7293 & $157 \mathrm{pc}$ & $9.77 \times 10^{44}$ & 906 & 1540 & 14900 \\
Solar Corona & 1 A.U. & $1.46 \times 10^{16}$ & 457 & 457 & 4420 \\
\hline \hline
\end{tabular}

* No extinction

We obtain the observed values for the electronic density, and the temperature of the Solar Corona $^{[12]}, \zeta=R / R_{\odot}$, where $R_{\odot}=6.96 \times 10^{10} \mathrm{~cm}$ is the solar radius

for $1 \leq \zeta \leq 3.83$

$$
n_{e}(\zeta)=1.55 \times 10^{8} \zeta^{-6} \times\left(1+1.93 \times \zeta^{-10}\right) \mathrm{cm}^{-3}
$$

$\&$ for $\zeta>3.83$

$$
\begin{gathered}
n_{e}(\zeta)=7.2 \times 10^{5} \zeta^{-2} \mathrm{~cm}^{-3} \\
T_{e}(\zeta)=\zeta^{-2 / 7} \times 10^{6} \mathrm{~K} .
\end{gathered}
$$

The number of signal photons $z_{P h_{s}}$ which arrive on the earth per $\mathrm{cm}^{2}$ per second would be:

$$
z_{P h_{s}}=\frac{Q(2 q)}{8 \pi L^{2}} \mathrm{~cm}^{-2} \mathrm{~s}^{-1},
$$

where $L$ is the distance to the object in $\mathrm{cm}$. The number of signal photons that can be detected by a receiver of diameter $\mathrm{D}$ will be:

$$
Z_{P h_{s}}=z_{P h_{s}} \times \frac{\pi D^{2}}{4}=\frac{Q(2 q)}{32 \cdot L^{2}} \times D^{2} \mathrm{~s}^{-1},
$$

$C(\Theta)$, the number of two-photon coincidences that can be detected with our receiver of diameter $D$ at a distance $L$ from the particular region, which depends on the subtended angle of the receiver

$$
\begin{gathered}
\Theta=\frac{D}{L} \text { radians. } \\
C(\Theta)=\frac{Q(2 q) D^{2}}{8 \pi L^{2}} \times \frac{1}{3}\left(\frac{D}{L}\right)^{2}=\frac{Q(2 q)}{24 \pi} \times\left(\frac{D}{L}\right)^{4} \\
D_{\text {min }}=\left[\frac{24 \pi \cdot C(\Theta)}{Q(2 q)}\right]^{1 / 4} \times L
\end{gathered}
$$

In table 1 we presented the minimum receiver diameters (including the effect of ISM extinction) for one two-photon coincidence per hour and per year for all the studied astrophysical objects in our paper ${ }^{[10]}$. 


\section{Conclusions}

- The possibility to observe this quantum effect in a naturally occurring astrophysical object seems particularly interesting. Since it would not only be observed outside of laboratory conditions, but the distance associated with the non-locality of such an entanglement would be unprecedented.

- The most feasible EPR Quantum Entanglement that can be detected in the Universe is the two-photon spontaneous transition of the hydrogen $2{ }^{2} S_{1 / 2}$ metastable level.

- The pair of photons keep their entanglement during their travel.

- The two-photon emission rate for Orion, IC 2149, NGC 7293, \& Sol.Cor. are $5.80 \times 10^{48}$, $9.39 \times 10^{45}, 9.77 \times 10^{44}, \& 1.46 \times 10^{16}$ pairs of photons per second respectively.

- The minimum sizes of the receiver diameters $D$ for one coincidence per year $272 \mathrm{~km}$ and $457 \mathrm{~km}$ for the Orion Nebula and Solar Corona respectively and 906 and $5200 \mathrm{~km}$ for NGC 7293 and IC 2149 respectively.

- The best astrophysical object to detect two-photon coincidences is the Solar Corona.

- Although we have explored the possibilities of the two-photon detection, we don't think that the proposed size level (about $457 \mathrm{~km}$ for the Solar Corona) could be realistically reached even in the long future.

\section{References}

[1] A. Aspect, J. Dalibard, \& G. Roger, Phys. Rev. Lett. 49 (1804)

[2] J. S. Bell, Physics 1 (195)

[3] D. Bohm, Quantum Theory Prentice-Hall, New York

[4] G. Breit, \& E. Teller, Ap. J. 91 (215)

[5] R. L. Brown, \& W. G. Mathews, Ap. J. 160 (939)

[6] J.F. Clauser, \& A. Shimony, Rep. Prog. Phys. 41 (1881)

[7] A. Einstein, B. Podolsky, \& N. Rosen, Phys. Rev. 47 (777)

[8] A. Fedrizzi et al., Nat. Phys. 5 (389)

[9] M. Göpert-Mayer Ann. Phys. 9 (273)

[10] J. Gomez, A, Peimbert, \& J. Echevarría, Optical Quantum Entanglement in Astrophysics, RMxAA45 (179)

[11] G. A. Gurzadyan PASP 88 (891)

[12] K. R. Lang, Astrophysical Formulae, Volumes I and II, 3rd Edition, Springer-Verlag, Berlin

[13] R. Novick, Physics of One and Two-Electron Atoms, ed F. Bopp, \& H. Kleinpoppen, North Holland, Amsterdam 
[14] D.E. Osterbrock, \& G.J. Ferland, Astrophysics of Gaseous Nebulae and Active Galactic Nuclei, 2nd. ed. University Press, Cambridge

[15] A. Peres, Quantum Theory: Concepts and Methods Kluwer, Dordrecht York

[16] L. Spitzer, \& J. Greenstein, Ap. J. 114 (407) 